\title{
Inhibition of Amyloid Fibrillization of Hen Egg-White Lysozymes by Rifampicin and $p$-Benzoquinone
}

\author{
Valerie H. Lieu, ${ }^{\dagger}$ Josephine W. Wu, ${ }^{\dagger}$ Steven S.-S. Wang, ${ }^{* \dagger}$ and Chia-Hung $\mathrm{Wu}^{\dagger}$ \\ Department of Chemical Engineering National Taiwan University, Taipei 10617, Taiwan, and Department of Optometry, Chung \\ Shan Medical University, Taichung 402, Taiwan
}

\begin{abstract}
It has been reported that more than 20 different human proteins can fold abnormally, resulting in the formation of pathological deposits and several lethal degenerative diseases. Despite extensive investigations on amyloid fibril formation, the detailed molecular mechanism remained rather elusive. The current research, utilizing hen egg-white lysozymes as a model system, is aimed at exploring inhibitory activities of two potential molecules against lysozyme fibril formation. We first demonstrated that the formation of lysozyme amyloid fibrils at $\mathrm{pH} 2.0$ was markedly enhanced by the presence of agitation in comparison with its quiescent counterpart. Next, via numerous spectroscopic techniques and transmission electron microscopy, our results revealed that the inhibition of lysozyme amyloid formation by either rifampicin or its analogue $p$-benzoquinone followed a concentration-dependent fashion. Furthermore, while both inhibitors were shown to acquire an anti-aggregating and a disaggregating activity, rifampicin, in comparison with $p$-benzoquinone, served as a more effective inhibitor against in vitro amyloid fibrillogenesis of lysozyme. It is our belief that the data reported in this work will not only reinforce the findings validated by others that rifampicin and $p$-benzoquinone serve as two promising preventive molecules against amyloid fibrillogenesis, but also shed light on a rational design of effective therapeutics for amyloidogenic diseases.
\end{abstract}

\section{Introduction}

The amyloidoses, a group of protein misfolding or conformational diseases, arise when a constituent protein or peptide undergoes a change in size or fluctuation in shape with resultant self-assembly and tissue deposition. Up to now, at least twenty different human proteins and peptides such as $\alpha$-synuclein, $\beta_{2^{-}}$ microglobulin, amylin, and $\beta$-amyloid have been isolated as the fibrillar components of disease-associated amyloid deposits (14). These amyloidogenic diseases individually have their unique pathological, clinical, and biochemical characteristics, and their corresponding amyloidogenic precursor proteins have unrelated functions and exhibit little sequence or structural homology. Despite extensive investigations on amyloid fibril formation, the detailed molecular mechanism remained largely unknown.

Aggregation/fibril formation is generally regarded to be associated with partially folded intermediate species that are susceptible to self-association due to the exposure of hydrophobic core (5). Amyloid is considered a general term delineating protein aggregates, and structures of amyloids are recognized to possess several tinctorial and physicochemical features in common: exhibition of $\beta$-sheet rich secondary structure, fibrillar morphology, birefringence upon staining with histological stain Congo red, insolubility in most solvents, and protease resistance. The pathological conformers resulted from an aggregation/ fibrillogenesis process in amyloidoses, which are rich in the content of cross- $\beta$ pleated sheet, and extensive protein fibrillization often occurs leading to an accumulation of the abnormally

* To whom correspondence should be addressed. Phone: 886-2-33665870. Fax: 886-2-2362-3040. E-mail: sswang@ntu.edu.tw.

† National Taiwan University.

$\doteqdot$ Chung Shan Medical University. folded proteins that correlate with cell death. The formation of these pathological conformers is influenced by genetic mutations, protein concentration, chaperones, and environmental factors $(6-9)$.

A growing number of evidence has indicated that amyloid fibrillization is not only possible to a certain group of proteins associated with amyloidoses. Nondisease-related proteins can be induced in vitro to polymerize into amyloid fibrils under certain favorable conditions such as heating, agitation, low $\mathrm{pH}$, pressure, and the presence of cosolvent $(10-14)$. The formation of fibrillar species in vitro from nondisease-associated proteins/ peptides is a theme that has been attracting renewed attention $(1,15,16)$.

Hen egg-white lysozyme (HEWL), an enzyme consisting of 129 amino acids that lyses the cell walls of bacteria, has been comprehensively studied. The lysozyme monomer has four disulfide bonds and adopts helix-rich conformation ( $\sim 30 \%$ $\alpha$-helix; $\sim 6 \% \beta$-sheet) (17). While only $\sim 40 \%$ of the hen eggwhite lysozyme is identical in sequence to the human lysozyme, these two forms of lysozymes, nevertheless, retain a high degree of structural homology. Recent studies have shown that, in addition to human lysozyme, several other variants of lysozymes are also capable of producing fibrillar species that exhibit characteristics of amyloid fibrils resulted from the group of proteins related to clinical amyloidoses $(18-20)$.

While amyloidoses have been the center of intense research efforts, no real cure is currently available toward treating the diseases. Given the situation/condition that no real cure is currently available toward treating the amyloidoses, inhibiting the production of pathological fibrillar/aggregated conformers or capturing these species has been by far envisaged as an effective approach to tackling amyloidoses. Extensive efforts 
have been directed toward seeking or developing anti-amyloidogenic/anti-aggregating agents as potential strategies to battle amyloidoses $(21,22)$. Evidence that rifampicin and $p$-benzoquinone prevented the aggregation of $\beta$-amyloid, amylin, and $\alpha$-synuclein $(23-25)$ prompted us to further examine their influence on other proteins, such as the fibrillogensis of HEWLs. Herein, via a number of spectroscopic techniques and transmission electron microscopy, we demonstrate that rifampicin and its derivative, $p$-benzoquinone, possess anti-aggregating and antiamyloidogenic activities, which were found to be dependent upon their concentrations and incubation time. Furthermore, experiments with inhibitors added at later times showed that both inhibitors possessed disaggregating activity against preformed HEWL fibrils. Taken together, our results indicated that rifampicin served as a more effective inhibitor in comparison with $p$-benzoquinone against amyloid fibrillogenesis of HEWLs. We believe that the data reported in this work will not only reinforce the findings validated by others that rifampicin and $p$-benzoquinone serve as two promising preventive molecules against amyloid fibrillogenesis, but also shed light on a rational design of potential therapeutics for amyloidogenic diseases.

\section{Materials and Methods}

Proteins and Reagents. Hen egg-white lysozyme (HEWL; EC 3.2.1.17), purchased from Merck (Germany), was refrigerated on receipt and used without further purification. Hydrochloric acid $(\mathrm{HCl})$ and dipotassium hydrogen phosphate $\left(\mathrm{K}_{2-}\right.$ $\mathrm{HPO}_{4}$ ) were obtained from Merck (Germany). Potassium dihydrogen phosphate $\left(\mathrm{KH}_{2} \mathrm{PO}_{4}\right)$ and disodium hydrogen phosphate $\left(\mathrm{Na}_{2} \mathrm{HPO}_{4}\right)$ were purchased from Showa Chemical, Co., Ltd (Japan). All other chemicals, unless otherwise specified, were obtained from Sigma Chemical Co. (St. Louis, MO).

Lysozyme Sample Solution Preparation. Sample solutions of $2 \mathrm{mg} / \mathrm{mL}$ HEWL were prepared by dissolving $0.1 \mathrm{~g}$ lyophilized HEWL in $50 \mathrm{~mL}$ of hydrochloric acid ( $\mathrm{pH}$ 2.0) with salts $(136.7 \mathrm{mM} \mathrm{NaCl}, 2.68 \mathrm{mM} \mathrm{KCl})$. HEWL sample solutions were first mixed via vortexing and then incubated in a reciprocating shaker bath, with a rotation rate of $30 \mathrm{rpm}$, under conditions of $55^{\circ} \mathrm{C}$ or $45^{\circ} \mathrm{C}$, during the course of the fibril formation/aggregation process.

Thioflavin T Fluorescence (ThT) Assay. Phosphate buffered saline (PBS) with $0.01(\mathrm{w} / \mathrm{v}) \%$ sodium azide was used to dissolve thioflavin $\mathrm{T}$ (ThT) to a final concentration of $10 \mu \mathrm{M}$. Lysozyme samples $(40 \mu \mathrm{L})$ taken at different times were added to $960 \mu \mathrm{L}$ of thioflavin T (ThT) solution and briefly mixed with vortex. ThT fluorescence intensity measurements were performed by exciting samples at $440 \mathrm{~nm}$ and recording emission intensities at $485 \mathrm{~nm}$ using an F-2500 Fluorescence Spectrophotometer (Hitachi, Japan). All measurements were taken in triplicate.

Circular Dichroism Spectroscopy. Circular dichroism (CD) spectra of lysozyme samples were recorded on a JASCO J-715 (150-S Type) spectrometer (Sunway Scientific Corporation) at $25{ }^{\circ} \mathrm{C}$ using a bandwidth of $2.0 \mathrm{~nm}$ with step interval of 0.1 $\mathrm{nm}$ and an averaging time of $2 \mathrm{~s}$. A $0.1 \mathrm{~cm}$ quartz cell was used for far-UV (200-260 nm) measurements. Three scans each of duplicate samples were measured and averaged. Control buffer scans were run in duplicate, averaged, and then subtracted from the sample spectra. The results were plotted as ellipticity (millidegree) versus wavelength ( $\mathrm{nm}$ ).

1-Anilinonaphthalene-8-sulfonic Acid (ANS)-Binding Assay. Lysozyme samples $(100 \mu \mathrm{L}$; concentration $=2 \mathrm{mg} / \mathrm{mL})$ were mixed with $900 \mu \mathrm{L}$ of $10 \mu \mathrm{M}$ 1-anilinonaphthalene-8sulfonic acid (ANS) dye in PBS with $0.01(\mathrm{w} / \mathrm{v}) \%$ sodium azide and then incubated in the dark for $30 \mathrm{~min}$ at room temperature. ANS fluorescence intensity measurements of mixtures taken at different times were performed by exciting samples at $380 \mathrm{~nm}$ and recording emission intensities from $420 \mathrm{~nm}$ to $580 \mathrm{~nm}$ using an F-2500 fluorescence spectrophotometer (Hitachi, Japan). Slits were adjusted to 5 and $10 \mathrm{~nm}$ for excitation and emission, respectively. All measurements were repeated at least three times. The representative ANS fluorescence intensity was taken at the average emission wavelength (AEW), which accounts for both changes in intensity and changes in the spectrum envelope. The determination of AEW was carried out using the following equation

$$
\mathrm{AEW}=\left(\sum F_{\mathrm{i}} \lambda_{\mathrm{i}}\right) /\left(\sum \lambda_{\mathrm{i}}\right)
$$

where $F_{\mathrm{i}}$ is the ANS fluorescence intensity at wavelength $\lambda_{\mathrm{i}}$. All measurements were taken in triplicate.

Transmission Electron Microscopy. A $10 \mu \mathrm{L}$ sample was placed on a carbon-stabilized, formvar-coated grid. Grids were negatively stained with $2 \%(\mathrm{w} / \mathrm{v})$ aqueous uranyl acetate (Electron Microscopy Sciences, Hatfield, PA) and then examined and photographed in a JEOL, JEM-1230EXII transmission electron microscope with Gantan Dual Vision CCD Camera (Tokyo, Japan) at an accelerating voltage of $100 \mathrm{kV}$.

Congo Red Binding Assay. To assess the presence of amyloid fibrils in the lysozyme solutions, Congo red binding studies were performed. Congo red dye was dissolved in PBS to a final concentration of $112 \mu \mathrm{M}$. Congo red absorbances of lysozyme sample solutions and the free dye controls were determined by adding Congo red to a final concentration of 18 $\mu \mathrm{M}$ and acquiring spectral measurements from 400 to $700 \mathrm{~nm}$ at $25{ }^{\circ} \mathrm{C}$ using a Spectronic Genesys 5 spectrophotometer (Spectronic Instrument, U.S.A.) (26). Both the lysozyme solutions and the control solutions were allowed to interact with Congo red for at least $30 \mathrm{~min}$ prior to recording their spectra. All measurements were taken in triplicate.

Data Evaluation of Amyloid Formation Kinetics. The data from ThT fluorescence measurements were fitted by a sigmoidal curve described by the following equation

$$
F=F_{i}+m_{i} t+\frac{F_{f}+m_{f} t}{1+e^{-\left[\left(t-t_{0}\right) / \tau\right]}}
$$

where $F$ is the fluorescence intensity at time $t, F_{i}$ the initial fluorescence intensity, $F_{f}$ the final fluorescence intensity, $t$ the incubation time, and $t_{0}$ the time to reach $50 \%$ of maximal fluorescence. The apparent rate constant for fibril growth is given by $1 / \tau$, and the lag time is determined to be $t_{0}-2 \tau$.

\section{Results}

Effect of Agitation on Kinetics of HEWL Amyloid Fibrillization. ThT is believed to interact rapidly and specifically with amyloid fibrils (27). Amyloid fibrillogenesis was widely detected by the ThT fluorescence assay, in which the increase in ThT fluorescence signal has been reported as an important indicator of the presence of amyloid fibril (27). As depicted in the inset of Figure 1A, in the absence of agitation, no significant ThT fluorescence emission was observed until $\sim 10$ days after the initiation of amyloid fibril formation at $55^{\circ} \mathrm{C}$. However, when dissolved in hydrochloric acid ( $\left.\mathrm{pH} 2.0\right)$ with $30 \mathrm{rpm}$ of agitation at $55^{\circ} \mathrm{C}$, the HEWL solution was observed to rapidly become visibly turbid and exhibited a dramatic enhancement in ThT fluorescence intensity within $\sim 2$ days in comparison with its quiescent counterpart. To better 

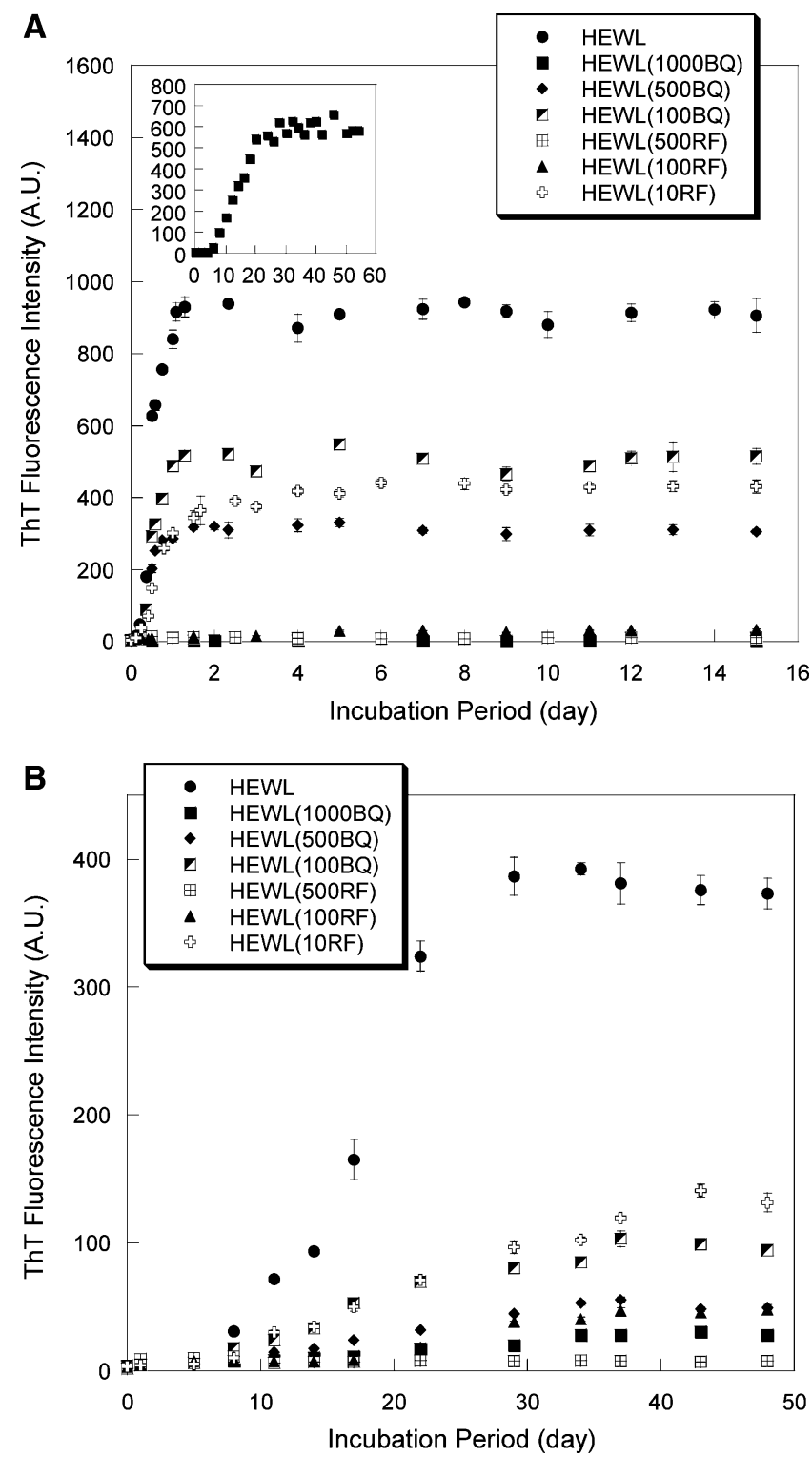

Figure 1. The effect of rifampicin or $p$-benzoquinone on the kinetics of amyloid fibril formation of hen egg-white lysozyme at (A) $55^{\circ} \mathrm{C}$ and (B) $45^{\circ} \mathrm{C}$ with agitation at $30 \mathrm{rpm}$. The extent of fibril formation was measured via ThT fluorescence as a function of incubation time of fibril formation process. The concentrations of rifampicin used were 10,100 , and $500 \mu \mathrm{M}$, and the concentrations of $p$-benzoquinone used were 100, 500, and $1000 \mu \mathrm{M}$. The inset of Figure 1A depicts the kinetics of amyloid fibril formation of hen egg-white lysozyme at $55^{\circ} \mathrm{C}$ without agitation. The $Y$ - and $X$-axes represent ThT fluorescence and incubation time, respectively. Each point represents the average of at least five independent measurements $(n \geq 5)$.

appraise the effectiveness of inhibitors, the HEWL samples were agitated in the following experiments.

Ample evidence suggests that the nucleation-based polymerization model has been widely employed to analyze phenomena or behaviors with a sigmoidal time-course response curve, in particular, the amyloid fibril formation process $(28-32)$. The two-phase nucleation-dependent polymerization behavior with sigmoidal time-course response, sufficiently characterized by two key parameters, the lag time of nucleation phase, and the growth rate of elongation phase was utilized to fit ThT fluorescence data $(33,34)$ (the fitting equation is described in the Materials and Methods section). The lag time and the apparent rate constant of fibril growth for HEWL at $55{ }^{\circ} \mathrm{C}$ without agitation were determined to be 5.55 day and 0.26 day $^{-1}$, respectively, while 0.27 day and 9.20 day $^{-1}$ for the case with agitation at $30 \mathrm{rpm}$. Evidently, the presence of agitation elevated the growth rate by about 35 -fold and considerably shortened the duration of lag phase prior to the rapid fibril formation.

Effects of Rifampicin and $p$-Benzoquinone on HEWL Fibril Formation. We first examined the influence of temperature on the progression of HEWL fibril formation. As illustrated in Figure 1A,B, the ThT fluorescence intensities of HEWLs with no inhibitors at 55 and $45^{\circ} \mathrm{C}$ climbed up and reached the maximum within $\sim 2$ and $\sim 30$ days, respectively. In addition, the maximum fluorescence signal of $\mathrm{HEWL}$ at $55^{\circ} \mathrm{C}$ is more than twice that at $45^{\circ} \mathrm{C}$ (see Figure 1A,B), implying that more HEWL fibrils were acquired at $55^{\circ} \mathrm{C}$. Similar experiments were also performed at a lower temperature of $37^{\circ} \mathrm{C}$. No perceptible elevation in fluorescence intensity was observed even after 55 days of incubation (data not shown).

In exploring if rifampicin or its analogue $p$-benzoquinone exerted an inhibitory activity against the formation of HEWL fibrils at $\mathrm{pH}$ 2.0, we monitored the changes of ThT fluorescence intensity at various concentrations of inhibitors as a function of incubation period. We show in Figure 1A,B that both rifampicin and $p$-benzoquinone at 55 or $45{ }^{\circ} \mathrm{C}$ retarded HEWL aggregation/fibril formation in a concentration-dependent fashion. The inhibitory effect was found to drop as the concentration of $p$-benzoquinone decreased from $1000 \mu \mathrm{M}$ to $100 \mu \mathrm{M}$, and near-maximal inhibition was achieved with $p$-benzoquinone at $1000 \mu \mathrm{M}$ regardless of the temperature used $(99.7 \%$ or $92.5 \%$ decrease of ThT fluorescence relative to that of HEWL alone at 55 or $45{ }^{\circ} \mathrm{C}$, respectively). Approximately 3-4 days after the outset of incubation at $55^{\circ} \mathrm{C}$, co-incubation of HEWL with rifampicin at $10 \mu \mathrm{M}, 100 \mu \mathrm{M}$, or $500 \mu \mathrm{M}$ was accompanied by $\sim 53.2 \%, \sim 96.7 \%$, or $\sim 98.9 \%$ reduction in ThT fluorescence emission, respectively. When incubated at $45^{\circ} \mathrm{C}$, the amyloid fibril-induced ThT fluorescence signal at $45^{\circ} \mathrm{C}$ was decreased by rifampicin from $97.9 \%(500 \mu \mathrm{M})$ to $65.8 \%(10 \mu \mathrm{M})$. Our aforementioned results suggest that the best anti-amyloidogenic potency was obtained with rifampicin in comparison with p-benzoquinone.

Effects of Rifampicin and $p$-Benzoquinone on HEWL Conformation. To retrieve insights regarding the molecular mechanism of inhibition of HEWL fibrillogenesis by the inhibitors, the CD spectroscopy was undertaken to follow the structural property, the secondary structure in particular, as aggregation/fibrillization proceed. HEWL solutions with or without inhibitory agents were subjected to far-UV CD analysis, and the time course of changes in the far-UV CD spectra of samples at various inhibitor concentrations were determined. Initially, the far-UV CD spectra of HEWL solutions without and with inhibitors all displayed a similar spectrum with a shoulder at $\sim 222 \mathrm{~nm}$ and an absorption minimum at $\sim 208 \mathrm{~nm}$, reflecting an $\alpha$-helix-rich conformation (see Figure 2A,B). Nevertheless, after aggregating for $\sim 3-4$ days at $55{ }^{\circ} \mathrm{C}$ or 29-30 days at $45^{\circ} \mathrm{C}$, an occurrence of structural transition ( $\alpha$ to $\beta$ transition) along with a striking alteration in the relative proportion of secondary structures was perceived for HEWL alone (CD spectra not shown). The CD spectra obtained from HEWL were observed to exhibit a characteristic pattern of $\beta$-sheet-rich conformation, with an absorption minimum around $216 \mathrm{~nm}$, clearly implying that the conformation of HEWL species was predominately $\beta$-structure. As depicted in Figures 2 and 3 , relative to the $\beta$-sheet-rich HEWL conformer, a variation in the shape of the $\mathrm{CD}$ spectrum was detected upon the addition of inhibitor at 55 or $45{ }^{\circ} \mathrm{C}$. A closer look at the $\mathrm{CD}$ spectra obtained at $55^{\circ} \mathrm{C}$ revealed that a slight difference 

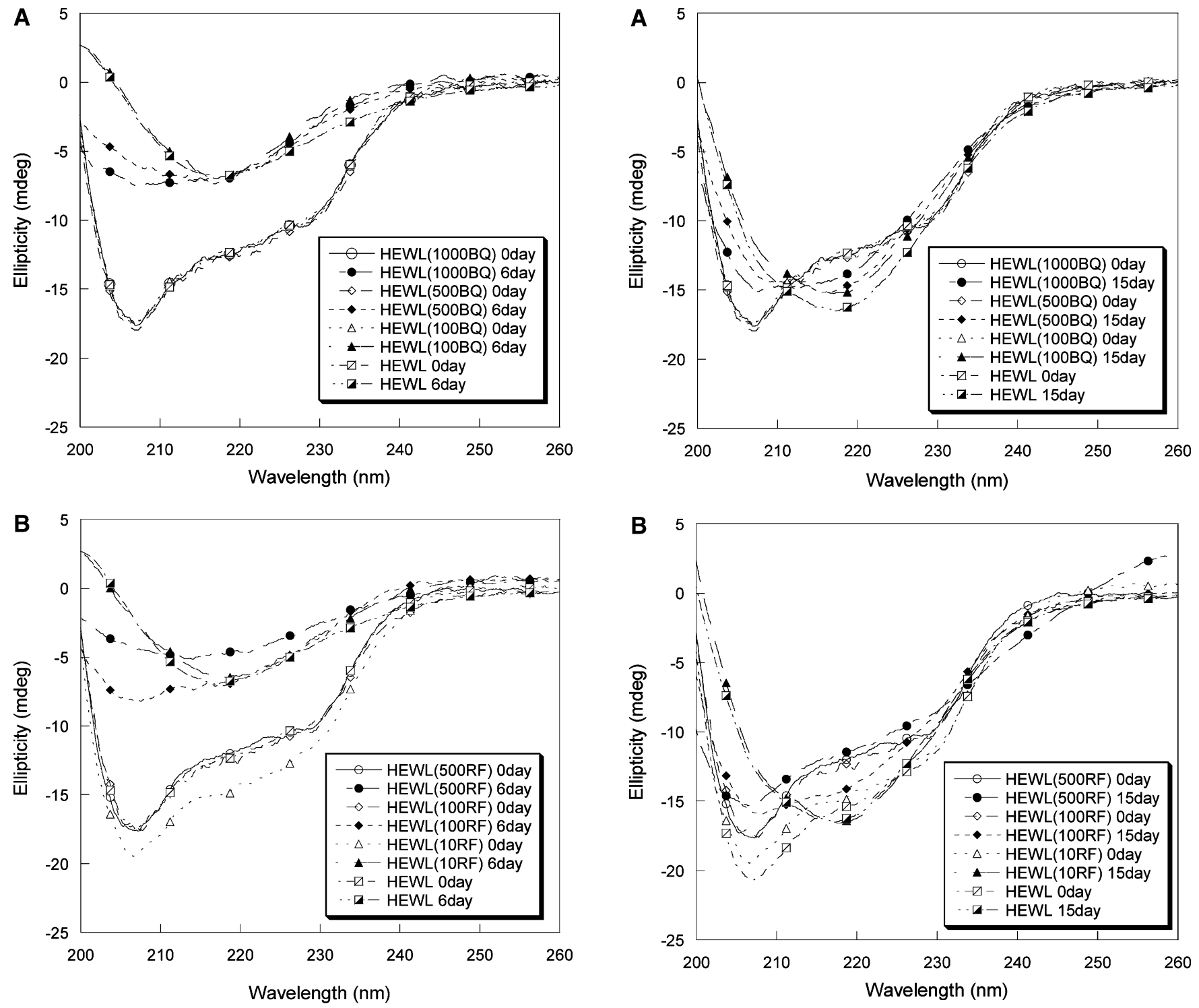

Figure 2. (A) Representative CD spectra of hen egg-white lysozyme with different concentrations of $p$-benzoquinone $(100,500$, and 1000 $\mu \mathrm{M})$ at $55^{\circ} \mathrm{C}$. (B) Representative CD spectra of hen egg-white lysozyme with different concentrations of rifampicin $(10,100$, and $500 \mu \mathrm{M})$ at $55^{\circ} \mathrm{C}$. Hen egg-white lysozymes were dissolved in hydrochloric acid (pH 2.0) with salt and the fibril formation process was accompanied with agitation at $30 \mathrm{rpm}$.

of spectrum was seen in HEWL samples plus lower concentration of $p$-benzoquinone $(100 \mu \mathrm{M})$ or rifampicin $(10 \mu \mathrm{M})$. Furthermore, this spectral variation became more dramatic as the concentration of either one of the inhibitors increased. As shown in Figure 2A,B that the negative peak in CD spectra at $\sim 218 \mathrm{~nm}$ disappeared when HEWLs incubated with $1000 \mu \mathrm{M}$ p-benzoquinone or $500 \mu \mathrm{M}$ rifampicin after 6 days of the process. Obviously, the presence of $p$-benzoquinone at 1000 $\mu \mathrm{M}$ or rifampicin at $500 \mu \mathrm{M}$ markedly prevented a structural transition from the native $\alpha$-helix-rich HEWL conformer to amyloidogenic $\beta$-sheet-rich species. A similar trend was also observed at later incubation times in the case of $45^{\circ} \mathrm{C}$ while lower ellipticity signals were seen in the $\mathrm{CD}$ spectra relative to the one at $55^{\circ} \mathrm{C}$ (see Figures $3 \mathrm{~A}$ and $3 \mathrm{~B}$ ). Our results indicated that the inhibitory potency possessed by these two inhibitors against the formation of $\beta$-sheet-rich HEWL conformation was in a concentration-dependent manner.

Effects of Rifampicin and $p$-Benzoquinone on HEWL Structure. To elucidate the effect of inhibitors on the changes

Figure 3. (A) Representative CD spectra of hen egg-white lysozyme with different concentrations of $p$-benzoquinone $(100,500$, and 1000 $\mu \mathrm{M}$ ) at $45^{\circ} \mathrm{C}$. (B) Representative CD spectra of hen egg-white lysozyme with different concentrations of rifampicin $(10,100$, and $500 \mu \mathrm{M})$ at $45^{\circ} \mathrm{C}$. Hen egg-white lysozymes were dissolved in hydrochloric acid $(\mathrm{pH} 2.0)$ with salt and the fibril formation process was accompanied with agitation at $30 \mathrm{rpm}$.

in tertiary structure of HEWL, we analyzed the ANS fluorescence emitted at average emission wavelength upon excitation at $380 \mathrm{~nm}$ with incubation time. The ANS fluorescent dye has been frequently used to probe for conformational properties, hydrophobicity in particular, in proteins $(35-38)$. It has been shown that, upon preferential binding to the hydrophobic surfaces, the fluorescence intensity induced by ANS enhances accompanying a blue shift in the wavelength of the emission maximum, owing to the extent of the nonpolar nature of the microenvironment of protein. We demonstrate in Figure 4A,B that the incubation of HEWL in the absence of inhibitor led to a pronounced enhancement in ANS fluorescence intensity as well as a drastic blue shift in the wavelength of fluorescence maximum, suggesting that HEWLs existed in a partially folded state with extensive solvent-exposed hydrophobic surfaces. For instance, ANS fluorescence signals were observed to be above 6000 units after 2 days of incubation at $55^{\circ} \mathrm{C}$ and after 28 days of incubation at $45^{\circ} \mathrm{C}$. However, in comparison with HEWLs alone, fairly lower fluorescence intensities of ANS were emitted 

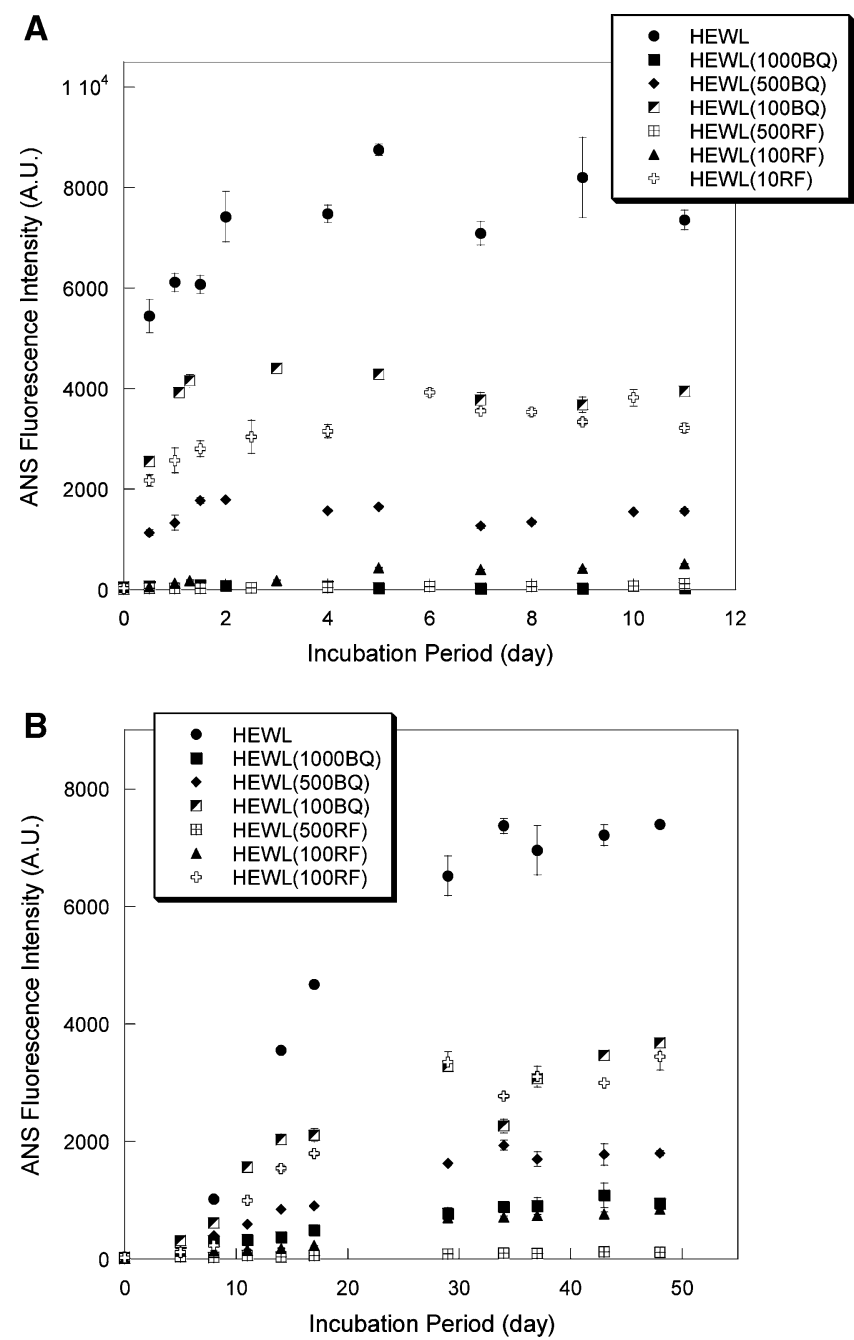

Figure 4. The effect of rifampicin or $p$-benzoquinone on the hydrophobicity of hen egg-white lysozyme during the course of aggregation at (A) $55^{\circ} \mathrm{C}$ and (B) $45^{\circ} \mathrm{C}$. The time-course of hydrophobic property of hen egg-white lysozyme was measured by ANS-binding fluorescence at different incubation times. Data were presented as an ANS fluorescence intensity taken at the average emission wavelength. The concentrations of $p$-benzoquinone used were 100, 500, and 1000 $\mu \mathrm{M}$ and the concentrations of rifampicin used were 10,100 , and 500 $\mu \mathrm{M}$. Each point represents the average of at least five independent measurements $(n \geq 5)$.

upon the addition of $p$-benzoquinone or rifampicin irrespective of the temperature used, implying that the exposure of hydrophobic regions was hampered due to the presence of either inhibitor. It was also discovered from our data that the extent of ANS-binding-competent exposed hydrophobic surface was inversely positively correlated with the inhibitor concentration. Optimal reductions in fluorescence intensities emitted upon ANS binding at both temperatures were achieved when $p$-benzoquinone at $1000 \mu \mathrm{M}$ and rifampicin at $500 \mu \mathrm{M}$ were used, that is, the maximal percentage reductions in ANS fluorescence intensity were $99.7 \%$ at $55^{\circ} \mathrm{C}$ and $92.5 \%$ at $45^{\circ} \mathrm{C}$ by $1000 \mu \mathrm{M}$ p-benzoquinone and $98.9 \%$ at $55{ }^{\circ} \mathrm{C}$ and $97.9 \%$ at $45{ }^{\circ} \mathrm{C}$ by $500 \mu \mathrm{M}$ rifampicin.

Effects of Rifampicin and $p$-Benzoquinone on HEWL Morphology. The species formed from HEWL aggregation with and without inhibitors were morphologically analyzed through the transmission electron microscopy. Figure 5A,B,C shows the representative electron micrographs of HEWL alone, HEWL with $1000 \mu \mathrm{M} p$-benzoquinone, and HEWL with $500 \mu \mathrm{M}$ rifampicin, respectively, after incubation for about 2 days at $55^{\circ} \mathrm{C}$. The micrograph in Figure 5A confirms the formation of
A

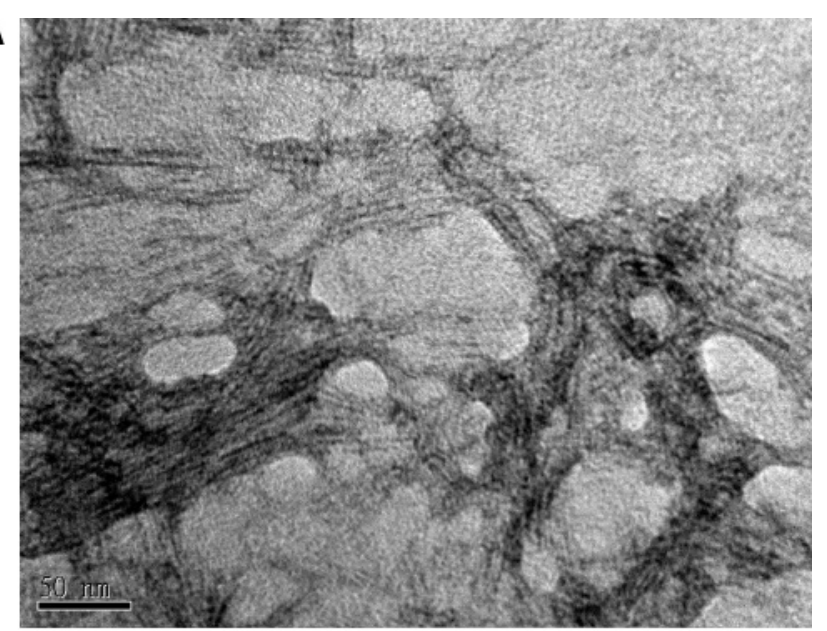

B

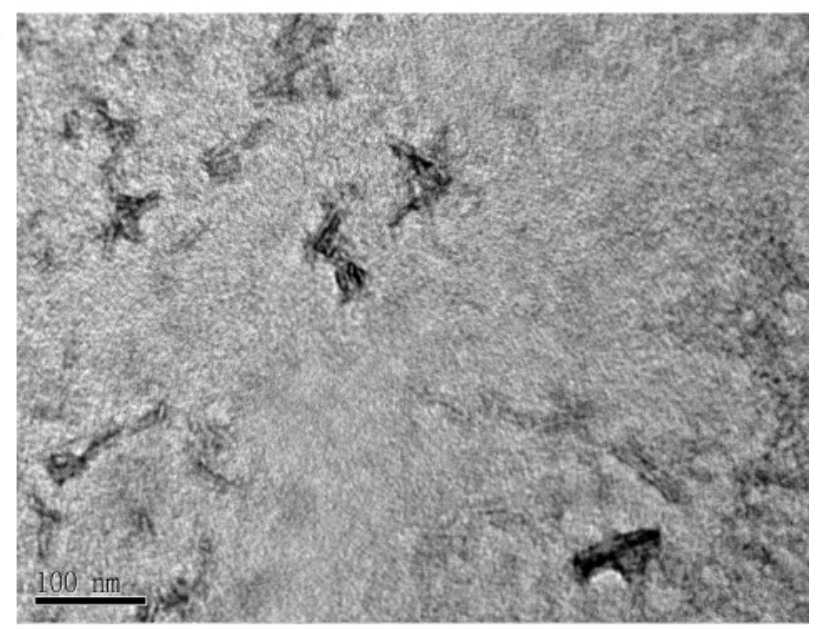

C

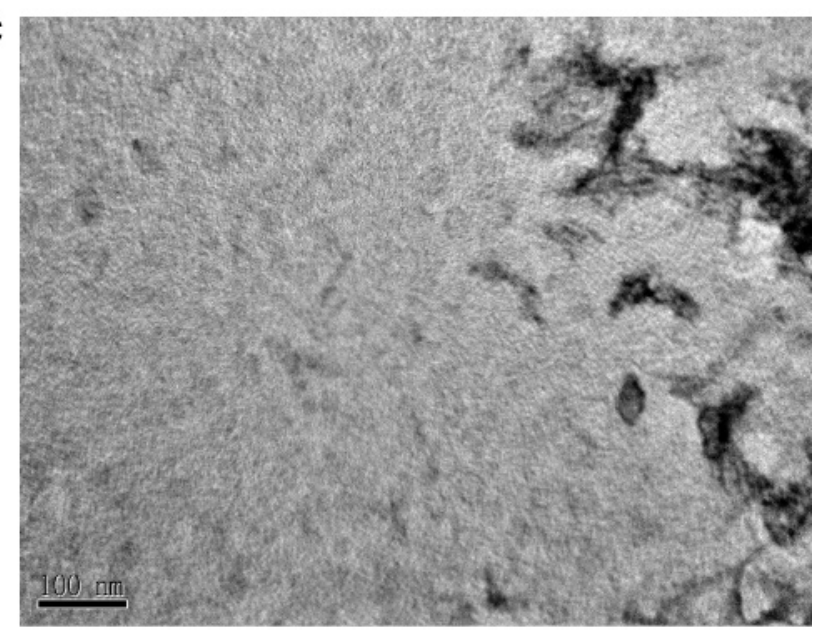

Figure 5. Electron micrographs of negatively stained hen egg-white lysozymes (A) without inhibitor after 2 days of aggregation; (B) with $1000 \mu \mathrm{M} p$-benzoquinone after 2 days of aggregation; and (C) with $500 \mu \mathrm{M}$ rifampicin after 2 days of aggregation. Lysozyme samples were prepared in hydrochloric acid ( $\mathrm{pH} 2.0)$ with salt and the fibril formation process was accompanied with agitation at $30 \mathrm{rpm}$.

amyloid fibrils/aggregated species in aged HEWL samples (2 days after the outset of fibrillization) in the absence of any inhibitor. No aggregated species/or fibrils were found in fresh samples of HEWL with or without inhibitors (micrographs not shown). Quantitatively, the aggregated/fibrillar HEWL species were noticeably absent from micrographs of HEWL-inhibitor mixtures (Figure 5B,C). Morphologically, as apposed to the cases in the presence of inhibitors, sheet-like structures with 
larger cross-sectional area composed of fibril bundles were discovered in HEWL fibril samples as seen in Figure 5A. Comparable micrographic results were obtained for HEWL samples with or without inhibitors after 2 days of incubation (pictures not shown).

Effects of Rifampicin and $p$-Benzoquinone on HEWL Disaggregation. To further assess the disaggregating abilities of both inhibitors, we next raised the question as to whether, once HEWL aggregation commenced, the addition of inhibitors could disaggregate the HEWL fibrils. We aggregated HEWLs under conditions analogous to those described previously and, at later time points, added the inhibitors at optimal concentrations determined from earlier experiments (rifampicin, $500 \mu \mathrm{M}$; $p$-benzoquinone, $1000 \mu \mathrm{M}$ ). The influence of the added inhibitors at $12 \mathrm{~h}$ after the launch of aggregation on HEWL fibrillogenesis is illustrated in Figure 6A. The effect of 1000 $\mu \mathrm{M}$ of $p$-benzoquinone on the HEWL solutions, in comparison to the solutions without inhibitors, yielded only $\sim 43 \%$ and $\sim 12 \%$ of ThT fluorescence intensity at 2 and 7 days, respectively, after the initiation of the aggregating process. However, with the addition of $500 \mu \mathrm{M}$ rifampicin, a substantial decline $(\geq 90 \%)$ in ThT signal was perceived 2 days after the onset of fibril formation and no further decrease was detected even after 11 days of incubation. Later addition of the inhibitors at $24 \mathrm{~h}$ after the start of aggregation was also tested (see Figure 6B). No significant difference was observed between the data for the addition of inhibitors at $12 \mathrm{~h}$ versus at $24 \mathrm{~h}$. We also utilized Congo red absorbance spectroscopy to probe for the presence of cross $\beta$-pleated sheet structure associated with amyloid fibrils. Protein solutions containing amyloid fibrils shifted the spectral properties of Congo red and exhibited a considerable increase in absorbance at around $540 \mathrm{~nm}$. As illustrated by the representative Congo red difference spectra in Figure 6C, the HEWLs alone after incubation for 9 days gave rise to a substantial rise in absorbance $(\sim 0.46)$ compared with that at the native state $(t=0)$. This is suggestive of the production of an appreciable amount of amyloid fibrils. Conversely, either one of the inhibitors, when added to HEWLs at $12 \mathrm{~h}$ or $24 \mathrm{~h}$ after the start of the aggregation process, attenuated the increase in absorbance intensity associated with HEWL fibril formation. Moreover, this Congo red binding result indicated that a loss in fibril content was observed in HEWLs under these conditions. Therefore, our results showed that both inhibitors possessed a disaggregating ability against the exiting HEWL aggregated species/fibrils. In addition, further confirmation that the rifampicin or $p$-benzoquinone-induced disaggregation of preformed HEWL fibrils came from electron microscopy analysis (micrographs not shown). Exposure of HEWL fibrils to either 500 $\mu \mathrm{M}$ rifampicin or $1000 \mu \mathrm{M} p$-benzoquinone resulted in a markedly reduced amount of fibrillar species. Taken altogether with our data from the previous sections, our findings evidently suggested that, in the range of concentrations used, rifampicin presented better anti-fibrillogenic/anti-aggregating and disaggregating properties than $p$-benzoquinone.

\section{Discussion}

The amyloidoses are complex, multiform disorders characterized by the polymerization of normally innocuous and soluble proteins/peptides into extracellular insoluble fibrils. More than 20 biochemically unique proteins have been isolated as the fibrillar components of disease-associated amyloid deposits ( $1-$ 4 ). While these amyloidogenic proteins share little sequence or structural homology, they all produce similar, straight, unbranched amyloid fibrils with characteristic cross $\beta$-sheet structure and the ability to bind Congo red $(8,39-41)$.
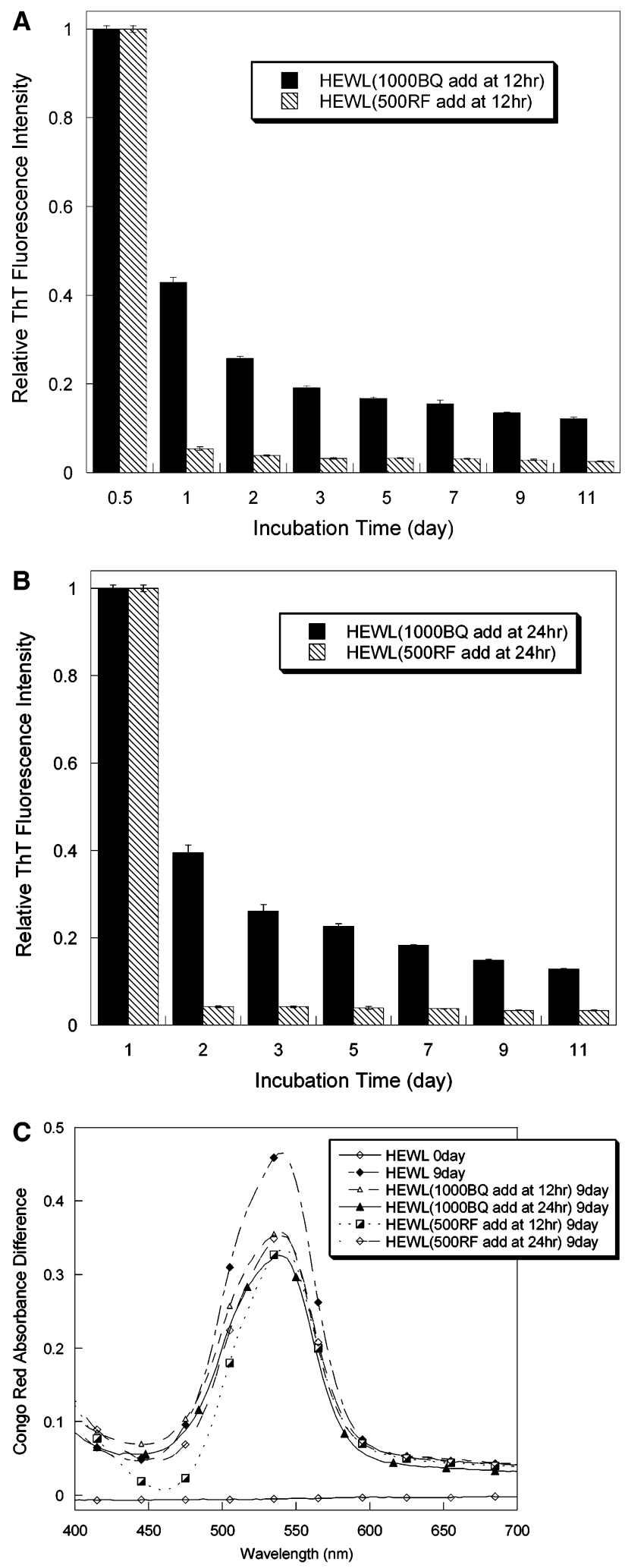

Figure 6. The disaggregating abilities of rifampicin and $p$-benzoquinone on hen egg-white lysozyme fibrils. The effect of inhibitors on hen egg-white lysozyme disaggregation was evaluated by ThT fluorescence analysis (A, B) and Congo red binding analysis (C). The relative ThT fluorescence intensity was defined as the ratio between ThT fluorescence signal with inhibitor addition and that without inhibitor at time t. Congo red difference spectrum was obtained by subtracting the buffer Congo red spectrum from sample Congo red binding spectrum. Rifampicin at $500 \mu \mathrm{M}$ or $p$-benzoquinone at $1000 \mu \mathrm{M}$ was added at 12 or $24 \mathrm{~h}$ after the launch of aggregation on HEWL fibrillogenesis. 
A variety of evidence suggests a strong connection between amyloid fibril formation and disease pathology. The amyloid fibrils or protofibrils derived from aggregated $\beta$-amyloid, $\beta_{2}$ microglobulin, atrial natriuretic peptide, and amylin have been demonstrated to be cytotoxic in vitro $(42-45)$. The cytotoxic effects of these proteins/peptides have been inhibited by compounds that bind to amyloid fibrils or inhibit fibril formation (46-49). Furthermore, interestingly, accumulating evidence has revealed that proteins unrelated to any amyloidogenic disease aggregate in vitro to form amyloid fibrillar species $(1,15,16)$.

A great body of evidence has suggested that reductions in the formation of fibrillar species as well as the amount of $\beta$-sheet structure contents have been considered as two promising therapeutic approaches in impeding the development of amyloidoses. A number of compounds have been found to retard the formation of aggregated or fibrillar species of amyloid proteins both in vitro and in vivo. For example, reports have demonstrated that various molecules such as alpha-crystallin Hsp20, nicotine, nordihydroguaiaretic acid, surfactants, and others delayed or prevented fibrillogenesis of amyloid proteins $(21,22,50)$.

Rifampicin is a semisynthetic bacteriocidal antibiotic derived from a form of rifamycin that is obtained from fermentation of Nocardia mediterranei. Rifampicin has been typically used to treat Mycobacterium infections such as leprosy and tuberculosis $(51,52)$. The action of rifampicin is through the inhibition of DNA-dependent RNA polymerase in bacterial cells by binding its $\beta$-subunit, thus preventing transcription of mRNA and subsequent translation to proteins $(51,52)$. Aside from these aforesaid properties, rifampicin has also been shown to exert inhibitory potency against the fibrillization/aggregation of a number of amyloid proteins/peptides such as $\beta$-amyloid peptide of $\mathrm{AD}$, amylin of type II diabetes mellitus, and $\alpha$-synuclein of Parkinson's disease $(23-25,53)$. Li and co-workers found that rifampicin not only inhibited fibrillization of $\alpha$-synuclein but also disintegrated formed fibrils in a dose-dependent fashion. The authors concluded that these inhibitory potencies were correlated with preferential stabilization of monomeric as well as oligomeric species (23). Via spectroscopic analyses and cytotoxicity assay, Tomiyama and co-workers proposed a rifampicin-induced inhibition against aggregation and neurotoxicity of $\beta$-amyloid peptides. In addition, this inhibitory activity was suggested to be highly pertinent to the scavenging ability of free radicals (25).

Previous studies demonstrated that rifampicin's analogue, $p$-benzoquinone, acquired the capability of intervening fibril formation or cytotoxicity induced by amyloid proteins/peptides. For instance, Tomiyama and co-workers, via the use of immunofluorescence microscopy, demonstrated that the protective action of $p$-benzoquinone against amylin fibril-induced toxicity was most likely attributed to the binding interaction between $p$-benzoquinone and amylin aggregated/fibrillar species (24). Moreover, $p$-benzoquinone has been reported to effectively attenuate fibrillogenesis and neurotoxic effect of $\beta$-amyloid peptides (25). Finally, with the aid of MS and NMR analyses, it has been shown that $p$-benzoquinone was able to suppress the aggregation process of $\alpha$-synuclein (54).

We first demonstrated that, in the absence of inhibitors, the rapid formation of HEWL fibrils was observed when the samples were agitated. We then tested rifampicin and $p$ benzoquinone for their effects on in vitro fibrillization of HEWL in the presence of agitation. In line with the results from the previous studies, the anti-amyloidogenic and anti-aggregating actions against HEWL fibrillar species were also revealed in our work. Reductions in fibril-induced ThT fluorescence signal as well as ANS fluorescence emission were noted upon preincubation with either one of the inhibitors and the declines were highly correlated with the concentration of inhibitors. Our CD measurements indicated that incubating HEWL with rifampicin or $p$-benzoquinone shifted the secondary structure to predominantly random coil structures and this structural transition was also observed to follow an inhibitor concentrationdependent manner. Furthermore, experiments using HEWLs treated with the inhibitors at later times of the process showed that both inhibitors presented a disaggregating activity against existing HEWL fibrils. This disaggregating property has also been pointed out in the study with $\alpha$-synuclein (23). Evidently, in the range of concentrations employed, our data revealed that rifampicin served as a more efficient anti-fibrillogenic/antiaggregating and disaggregating agent than $p$-benzoquinone.

It has been suggested that the presence of the naphthoquinone structure of rifampicin accounted for its inhibitory activity against $\beta$-amyloid peptide fibrillization (25); however, the detailed mechanisms of the inhibitory action against the aggregation or fibril formation of amyloid peptides/proteins exerted by rifampicin and its derivatives remain largely unknown. Further investigation is warranted to explore the underlying mechanism(s) of the interaction between HEWL and rifampicin or $p$-benzoquinone.

In summary, various spectroscopic techniques and transmission electron microscopy were used in this work to examine the anti-amyloidogenic and disaggregating effects of two potential inhibitory molecules, rifampicin and $p$-benzoquinone, on in vitro HEWL fibrillogenesis. Under the agitation condition, we have shown that rifampicin and $p$-benzoquinone exerted a dose-dependent inhibitory activity against $\beta$-sheet formation and aggregation, characteristics of amyloid fibril formation. Also, disaggregating abilities against preformed fibrils were found on both inhibitors. It is important to note that further research is warranted to examine the roles of rifampicin and $p$-benzoquinone in preventing HEWL fibrillogenesis. While both inhibitors perhaps serve as two model pharmacological agents and their inhibition on HEWL fibril formation might be somewhat different from that of the inhibitory action against fibril formation within the in vivo system, we believe that the outcome from this research will enable us, not only to comprehend the mechanism(s) of amyloid protein, diseaserelated or nondisease-related, self-association process, but also to help develop potential targets for molecular therapeutics in the prevention or delay of amyloid formation implicated in amyloidogenic diseases.

\section{Acknowledgment}

This work was supported by grants from the National Science Council, Taiwan. Authors would like to thank Dr. Rita P.-Y. Chen from the Institute of Biochemistry at Academia Sinica, Taiwan, for the assistance with CD measurements.

\section{References and Notes}

(1) Dobson, C. M. Principles of protein folding, misfolding and aggregation. Semin. Cell Dev. Biol. 2004, 15 (1), 3-16.

(2) Wang, S. S. S.; Good, T. A. An overview of Alzheimer's disease. J. Chin. Inst. Chem. Eng. 2005, 36 (6), 533-559.

(3) Ross, C. A.; Poirier, M. A. Protein aggregation and neurodegenerative disease. Nat. Med. 2004, 10 (Suppl), S10-S17.

(4) Uversky, V. N.; Fink, A. L. Conformational constraints for amyloid fibrillation: The importance of being unfolded. Biochim. Biophys. Acta 2004, 1698 (2), 131-153. 
(5) Oberg, K.; Chrunyk, B. A.; Wetzel, R.; Fink, A. L. Nativelike secondary structure in interleukin-1 beta inclusion bodies by attenuated total reflectance FTIR. Biochemistry 1994, 33 (9), 2628-2634.

(6) Carrell, R. W.; Gooptu, B. Conformational changes and diseaseSerpins, prions and Alzheimer's. Curr. Opin. Struct. Biol. 1998, 8 (6), 799-809.

(7) Ji, S. R.; Wu, Y.; Sui, S. F. Study of the correlation of secondary structure of beta-amyloid peptide (Abeta40) with the hydrophobic exposure under different conditions. Gen. Physiol. Biophys. 2002, $21(4), 415-427$.

(8) Lansbury, P. T., Jr. Evolution of amyloid: What normal protein folding may tell us about fibrillogenesis and disease. Proc. Natl. Acad. Sci. U.S.A. 1999, 96 (7), 3342-3344.

(9) Soto, C. Alzheimer's and prion disease as disorders of protein conformation: implications for the design of novel therapeutic approaches. J. Mol. Med. 1999, 77 (5), 412-418.

(10) Lai, Z.; Colon, W.; Kelly, J. W. The acid-mediated denaturation pathway of transthyretin yields a conformational intermediate that can self-assemble into amyloid. Biochemistry 1996, 35 (20), 64706482 .

(11) Sluzky, V.; Tamada, J. A.; Klibanov, A. M.; Langer, R. Kinetics of insulin aggregation in aqueous solutions upon agitation in the presence of hydrophobic surfaces. Proc. Natl. Acad. Sci. U.S.A. 1991, 88 (21), 9377-9381.

(12) Ferrao-Gonzales, A. D.; Souto, S. O.; Silva, J. L.; Foguel, D. The preaggregated state of an amyloidogenic protein: Hydrostatic pressure converts native transthyretin into the amyloidogenic state. Proc. Natl. Acad. Sci. U.S.A. 2000, 97 (12), 6445-6450.

(13) Sluzky, V.; Klibanov, A. M.; Langer, R. Mechanism of Insulin Aggregation and Stabilization in Agitated Aqueous-Solutions. Biotechnol. Bioeng. 1992, 40 (8), 895-903.

(14) Shtilerman, M. D.; Ding, T. T.; Lansbury, P. T., Jr. Molecular crowding accelerates fibrillization of alpha-synuclein: Could an increase in the cytoplasmic protein concentration induce Parkinson's disease? Biochemistry 2002, 41 (12), 3855-3860.

(15) Fandrich, M.; Forge, V.; Buder, K.; Kittler, M.; Dobson, C. M.; Diekmann, S. Myoglobin forms amyloid fibrils by association of unfolded polypeptide segments. Proc. Natl. Acad. Sci. U.S.A. 2003, 100 (26), 15463-15468.

(16) Kallberg, Y.; Gustafsson, M.; Persson, B.; Thyberg, J.; Johansson, J. Prediction of amyloid fibril-forming proteins. J. Biol. Chem. 2001, 276 (16), 12945-12950.

(17) Vaney, M. C.; Maignan, S.; Ries-Kautt, M.; Ducriux, A. Highresolution structure $(1.33 \mathrm{~A})$ of a HEW lysozyme tetragonal crystal grown in the APCF apparatus. Data and structural comparison with a crystal grown under microgravity from SpaceHab-01 mission. Acta Crystallogr., Sect. D: Biol. Crystallogr. 1996, 52 (Pt 3), 505-517.

(18) Cao, A.; Hu, D.; Lai, L. Formation of amyloid fibrils from fully reduced hen egg white lysozyme. Protein Sci. 2004, 13 (2), 319324.

(19) Frare, E.; Polverino, De Laureto, P.; Zurdo, J.; Dobson, C. M.; Fontana, A. A highly amyloidogenic region of hen lysozyme. J. Mol. Biol. 2004, 340 (5), 1153-1165.

(20) Fujiwara, S.; Matsumoto, F.; Yonezawa, Y. Effects of salt concentration on association of the amyloid protofilaments of hen egg white lysozyme studied by time-resolved neutron scattering. $J$. Mol. Biol. 2003, 331 (1), 21-28.

(21) Estrada, L. D.; Soto, C. Disrupting beta-amyloid aggregation for Alzheimer disease treatment. Curr. Top. Med. Chem. 2007, 7 (1), $115-126$.

(22) Porat, Y.; Abramowitz, A.; Gazit, E. Inhibition of amyloid fibril formation by polyphenols: Structural similarity and aromatic interactions as a common inhibition mechanism. Chem. Biol. Drug Des. 2006, 67 (1), 27-37.

(23) Li, J.; Zhu, M.; Rajamani, S.; Uversky, V. N.; Fink, A. L. Rifampicin inhibits alpha-synuclein fibrillation and disaggregates fibrils. Chem. Biol. 2004, 11 (11), 1513-1521.

(24) Tomiyama, T.; Kaneko, H.; Kataoka, K.; Asano, S.; Endo, N. Rifampicin inhibits the toxicity of pre-aggregated amyloid peptides by binding to peptide fibrils and preventing amyloid-cell interaction. Biochem. J. 1997, 322 ( Pt 3), 859-865.

(25) Tomiyama, T.; Shoji, A.; Kataoka, K.; Suwa, Y.; Asano, S.; Kaneko, H.; Endo, N. Inhibition of amyloid beta protein aggregation and neurotoxicity by rifampicin. Its possible function as a hydroxyl radical scavenger. J. Biol. Chem. 1996, 271 (12), 6839-6844.
(26) Klunk, W. E.; Pettegrew, J. W.; Abraham, D. J. Quantitative evaluation of congo red binding to amyloid-like proteins with a betapleated sheet conformation. J. Histochem. Cytochem. 1989, 37 (8), $1273-1281$.

(27) LeVine, H., III. Thioflavine T interaction with synthetic Alzheimer's disease beta- amyloid peptides: detection of amyloid aggregation in solution. Protein Sci. 1993, 2 (3), 404-410.

(28) Berlin, Y. A.; Burin, A. L.; Siebbeles, L. D. A.; Ratner, M. A. Conformationally gated rate processes in biological macromolecules. J. Phys. Chem. A 2001, 105 (23), 5666-5678.

(29) Nelson, J.; Chandler, R. E. Random walk models of charge transfer and transport in dye sensitized systems. Coord. Chem. Rev. 2004, 248 (13-14), 1181-1194.

(30) Kodaka, M. Requirements for generating sigmoidal time-course aggregation in nucleation-dependent polymerization model. Biophys. Chem. 2004, 107 (3), 243-253.

(31) Phillips, J. C. Stretched exponential relaxation in molecular and electronic glasses. Rep. Prog. Phys. 1996, 59 (9), 1133-1207.

(32) Ross, J.; Vlad, M. O. Nonlinear kinetics and new approaches to complex reaction mechanisms. Annu. Rev. Phys. Chem. 1999, 50, $51-78$.

(33) Harper, J. D.; Lansbury, P. T., Jr. Models of amyloid seeding in Alzheimer's disease and scrapie: Mechanistic truths and physiological consequences of the time-dependent solubility of amyloid proteins. Annu. Rev. Biochem. 1997, 66, 385-407.

(34) Nielsen, L.; Khurana, R.; Coats, A.; Frokjaer, S.; Brange, J.; Vyas, S.; Uversky, V. N.; Fink, A. L. Effect of environmental factors on the kinetics of insulin fibril formation: Elucidation of the molecular mechanism. Biochemistry 2001, 40 (20), 6036-6046.

(35) Semisotnov, G. V.; Rodionova, N. A.; Razgulyaev, O. I.; Uversky, V. N.; Gripas, A. F.; Gilmanshin, R. I. Study of the "molten globule" intermediate state in protein folding by a hydrophobic fluorescent probe. Biopolymers 1991, 31 (1), 119-128.

(36) Liu, C. P.; Li, Z. Y.; Huang, G. C.; Perrett, S.; Zhou, J. M. Two distinct intermediates of trigger factor are populated during guanidine denaturation. Biochimie 2005, 87 (11), 1023-1031.

(37) Smoot, A. L.; Panda, M.; Brazil, B. T.; Buckle, A. M.; Fersht, A. R.; Horowitz, P. M. The binding of bis-ANS to the isolated GroEL apical domain fragment induces the formation of a folding intermediate with increased hydrophobic surface not observed in tetradecameric GroEL. Biochemistry 2001, 40 (14), 4484-4492.

(38) Sirangelo, I.; Bismuto, E.; Tavassi, S.; Irace, G. Apomyoglobin folding intermediates characterized by the hydrophobic fluorescent probe 8-anilino-1-naphthalene sulfonate. Biochim. Biophys. Acta 1998, 1385 (1), 69-77.

(39) Glenner, G. G. Amyloid deposits and amyloidosis. The betafibrilloses (first of two parts). N. Engl. J. Med. 1980, 302 (23), 12831292.

(40) Glenner, G. G. Amyloid deposits and amyloidosis: the betafibrilloses (second of two parts). N. Engl. J. Med. 1980, 302 (24), 1333-1343

(41) Kelly, J. W. Alternative conformations of amyloidogenic proteins govern their behavior. Curr. Opin. Struct. Biol. 1996, 6 (1), 11-17.

(42) Rymer, D. L.; Good, T. A. The role of G protein activation in the toxicity of amyloidogenic Abeta-(1-40), Abeta- $(25-35)$, and bovine calcitonin. J. Biol. Chem. 2001, 276 (4), 2523-2530.

(43) Seilheimer, B.; Bohrmann, B.; Bondolfi, L.; Muller, F.; Stuber, D.; Dobeli, H. The toxicity of the Alzheimer's beta-amyloid peptide correlates with a distinct fiber morphology. J. Struct. Biol. 1997, 119 (1), 59-71

(44) Walsh, D. M.; Lomakin, A.; Benedek, G. B.; Condron, M. M.; Teplow, D. B. Amyloid beta-protein fibrillogenesis. Detection of a protofibrillar intermediate. J. Biol. Chem. 1997, 272 (35), 2236422372.

(45) Ward, R. V.; Jennings, K. H.; Jepras, R.; Neville, W.; Owen, D. E.; Hawkins, J.; Christie, G.; Davis, J. B.; George, A.; Karran, E. H.; Howlett, D. R. Fractionation and characterization of oligomeric, protofibrillar and fibrillar forms of beta-amyloid peptide. Biochem. J. 2000, 348 (Pt 1), 137-144.

(46) Burgevin, M. C.; Passat, M.; Daniel, N.; Capet, M.; Doble, A. Congo red protects against toxicity of beta-amyloid peptides on rat hippocampal neurones. Neuroreport 1994, 5 (18), 2429-2432.

(47) Lorenzo, A.; Yankner, B. A. Beta-amyloid neurotoxicity requires fibril formation and is inhibited by congo red. Proc. Natl. Acad. Sci. U.S.A. 1994, 91 (25), 12243-12247. 
(48) Pollack, S. J.; Sadler, II.; Hawtin, S. R.; Tailor, V. J.; Shearman, M. S. Sulfated glycosaminoglycans and dyes attenuate the neurotoxic effects of beta-amyloid in rat PC12 cells. Neurosci. Lett. 1995, 184 (2), 113-116.

(49) Sadler, II.; Smith, D. W.; Shearman, M. S.; Ragan, C. I.; Tailor, V. J.; Pollack, S. J. Sulphated compounds attenuate beta-amyloid toxicity by inhibiting its association with cells. Neuroreport 1995, 7 (1), 49-53.

(50) Lee, S.; Carson, K.; Rice-Ficht, A.; Good, T. Hsp20, a novel \{alpha\}-crystallin, prevents A $\{$ beta\} fibril formation and toxicity. Protein Sci. 2005, 14 (3), 593-601.

(51) Sensi, P. History of the development of rifampin. Rev Infect Dis. 1983, 3, 402-406.

(52) Senbaga, N.; Davies, E. M. Inadvertent intrathecal administration of rifampicin. Br. J. Clin. Pharmacol. 2005, 60 (1), 116.
(53) Tomiyama, T.; Asano, S.; Suwa, Y.; Morita, T.; Kataoka, K.; Mori, H.; Endo, N. Rifampicin prevents the aggregation and neurotoxicity of amyloid beta protein in vitro. Biochem. Biophys. Res. Commun. 1994, 204 (1), 76-83.

(54) Li, H. T.; Lin, D. H.; Luo, X. Y.; Zhang, F.; Ji, L. N.; Du, H. N.; Song, G. Q.; Hu, J.; Zhou, J. W.; Hu, H. Y.; Inhibition, of alphasynuclein fibrillization by dopamine analogs via reaction with the amino groups of alpha-synuclein. Implication for dopaminergic neurodegeneration. FEBS J. 2005, 272 (14), 3661-3672.

Received November 20, 2006. Accepted April 18, 2007.

BP060353N 